\title{
Investigation of TESPEL cloud dynamics in Wendelstein 7-X stellarator
}

\author{
G. Kocsis $^{1}$, N. Tamura ${ }^{2}$, R. Bussiahn ${ }^{3}$, K.J. McCarthy ${ }^{4}$, J. Baldzuhn ${ }^{3}$, C. Biedermann ${ }^{3}$, \\ G. Cseh ${ }^{1}$, H. Damm ${ }^{3}$, R. König ${ }^{3}$, N. Panadero ${ }^{4}$, T. Szepesi ${ }^{1}$ and the W7-X Team* \\ ${ }^{1}$ Wigner RCP RMI, Budapest, Hungary \\ ${ }^{2}$ National Institute for Fusion Science, National Institutes of Natural Sciences, Toki, Japan \\ ${ }^{3}$ Max Planck Institute for Plasma Physics, Greifswald, Germany \\ ${ }^{4}$ Laboratorio Nacional de Fusion, CIEMAT, Madrid, Spain
}

Cryogenic pellet injection is one of the prime candidates to fuel tokamaks and stellarators. Pellets are usually injected from the magnetic high-field side (HFS) of a tokamak since the $\operatorname{grad}(\mathrm{B})$ caused $\mathbf{E x B}$ drift pushes the pellet cloud towards the plasma center. This is favorable for deep particle deposition and higher fueling efficiency. In Wendelstein 7-X (W7-X) stellarator the magnetic geometry is not axial symmetric therefore the situation is more complex. Additionally, no clear advantage of HFS Hydrogen pellet injection is reported and cloud drifting was found not to be in the $\operatorname{grad}(\mathrm{B})$ direction [1].

A Tracer-Encapsulated Solid Pellet (TESPEL) [2] injection system was recently developed and installed at the W7-X [3]. TESPEL is injected radially from the low-field side (LFS) by a gas gun. A tracer impurity (typically a high-Z material like iron or tungsten) for impurity transport investigations is embedded into a polystyrene-polymer $\left(\mathrm{C}_{8} \mathrm{H}_{8}\right)_{n}$ sphere with an outer diameter of $<1 \mathrm{~mm}$. The polystyrene sphere has similar ablation characteristics to cryogenic H pellets [4] which makes it also useful for pellet cloud evolution and drift investigations. Moreover, the low order ionic states of carbon (C) can be easily distinguished by spectroscopic methods thereby allowing us to study both the neutral and the ionised parts of the pellet cloud. Accordingly, a fast framing camera, set to view from behind the TESPEL injection port, was installed on $\mathrm{W} 7-\mathrm{X}$ in order to investigate the polystyrene ablation, cloud formation, expansion and drift processes. Wavelength selection by interference filters was used to separate certain ionic species (538 nm (FWHM: 2.5 nm), $465 \mathrm{~nm}$ (FWHM: 2 nm) and $656 \mathrm{~nm}$ (FWHM: $\sim 3 \mathrm{~nm}$ ) for $\mathrm{C}, \mathrm{C}^{2+}$ and $\mathrm{H}$, respectively). Due to the wavelength width of the $\mathrm{H}_{\alpha}$ filter some $\mathrm{C}^{+}$radiation may interfere. With careful Region-of-Interest (ROI) selection on the detector chip the temporal resolution of the system could be enhanced up to $2 \mu \mathrm{s}$.

In the last experimental campaign, the TESPEL injector was commissioned and could inject one TESPEL per discharge. Movies were recorded with the fast camera; the filter was changed 
on a shot-by-shot basis. This observation view allows us to determine the vertical and poloidal movement of the TESPEL, and to investigate the radiation distribution of the polystyrene cloud along and perpendicular to the magnetic field lines. Typical short exposure $\left(\mathrm{C}: 1.9 \mu \mathrm{s}, \mathrm{C}^{2+}: 0.4\right.$ $\mu$ s per frame, $525 \mathrm{kHz}$ frame rate for both) images about $\mathrm{C}$ and $\mathrm{C}^{2+}$ radiation distributions are plotted in Fig.1. From such snapshots the position of the TESPEL (i.e. at the location of maximum radiation for $\mathrm{C}$ ) was determined during its ablation, showing that the TESPEL follows the designated radial trajectory, i.e., no vertical and poloidal deflection was seen.
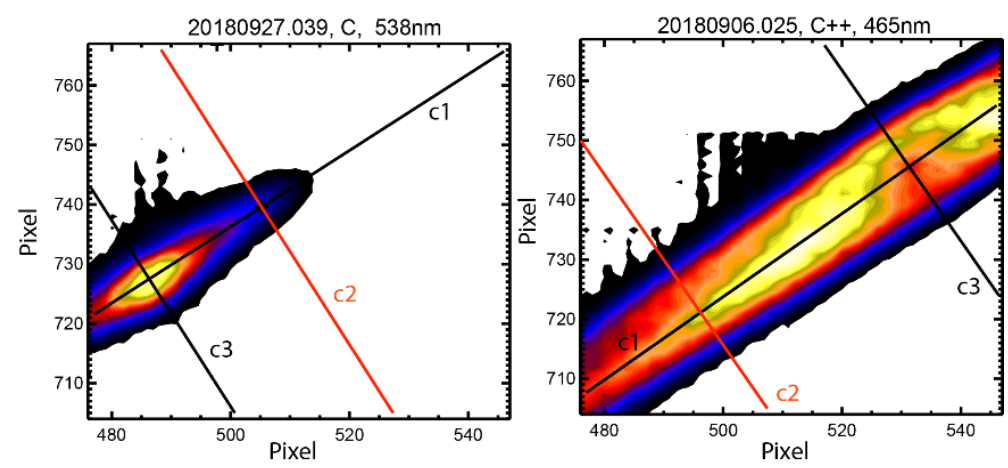

Figure 1. Short exposure images of $\mathrm{C}$ (left) and $\mathrm{C}^{2+}$ (right) radiation during TESPEL ablation. The $\mathrm{c} 1$ line represents the magnetic field line crossing the centre of the cloud where the location of TESPEL is expected. The c3 line is perpendicular to the field line and also crosses the expected TESPEL position. $\mathrm{c} 2$ is also perpendicular to the field line but selected to be further away from the TESPEL. c1, c2 and c3 are used to calculate the line cuts (intensity profiles) plotted in Figs. 2 and 3.

In order to visualise the distribution and evolution of the TESPEL cloud, intensity profiles along the magnetic field line $(\mathrm{B} \|)$ crossing the centre (c1, see Fig.1) of the cloud and perpendicular to the magnetic field line $(\mathrm{B} \perp)$ at two positions (at the centre: $\mathrm{c} 3$ and further away: c2) were calculated. Fig. 2 and Fig. 3 summarize these intensity profiles for $\mathrm{C}$ and $\mathrm{C}^{2+}$, respectively. Here both the individual profiles for consecutive frames and the radiation distributions as a function of time and distance along the cut lines are plotted. The details of the plotted figures can be found in the caption of Fig. 2. Analysing Fig. 2 and Fig. 3 the following conclusions can be drawn.

For neutral carbon the cloud is slightly elongated along the magnetic field line with a $\mathrm{B} \perp$ size of about $1 \mathrm{~cm}$ and a B\| size of about $1-2 \mathrm{~cm}$ (see Fig 2.d-f). The distribution is Gaussian-like, having one maximum. The $\mathrm{C}$ cloud line radiation is proportional to the product of the carbon density (dropping with distance measured from the pellet surface, determined by the spherical expansion of the neutral cloud) and the local target plasma electron density (the cloud electron 
density is low in this region - as long as the cloud is neutral) confirming the measured Gaussian-like radiation distribution.

Since cloud ions are streaming both parallel and anti-parallel to $\mathrm{B}$ the $\mathrm{C}^{2+}$ cloud (plasmoid) is strongly elongated along the magnetic field line with $\mathrm{B} \perp$ size of about $1-2 \mathrm{~cm}$ and $\mathrm{B} \|$ size of 5-20 cm. The cloud has a double peaked structure along the magnetic field line (see fig.3.d). The reason for this structure is that the $\mathrm{C}^{2+}$ radiation is probably dominated by the electron impact excitation of the pellet cloud electrons, which have orders of magnitude higher density than target plasma electrons at least at the cloud regions where $\mathrm{C}^{2+}$ density is high. Additionally, both the $\mathrm{C}^{2+}$ and the cloud electron density distributions have a maximum along the magnetic field line symmetrically on both sides of the pellet, resulting in the double-peaked structure. These features of the neutral and ionized cloud (plasmoid) part of the TESPEL are similar to the behaviors of the $\mathrm{H}$ pellet cloud [5].
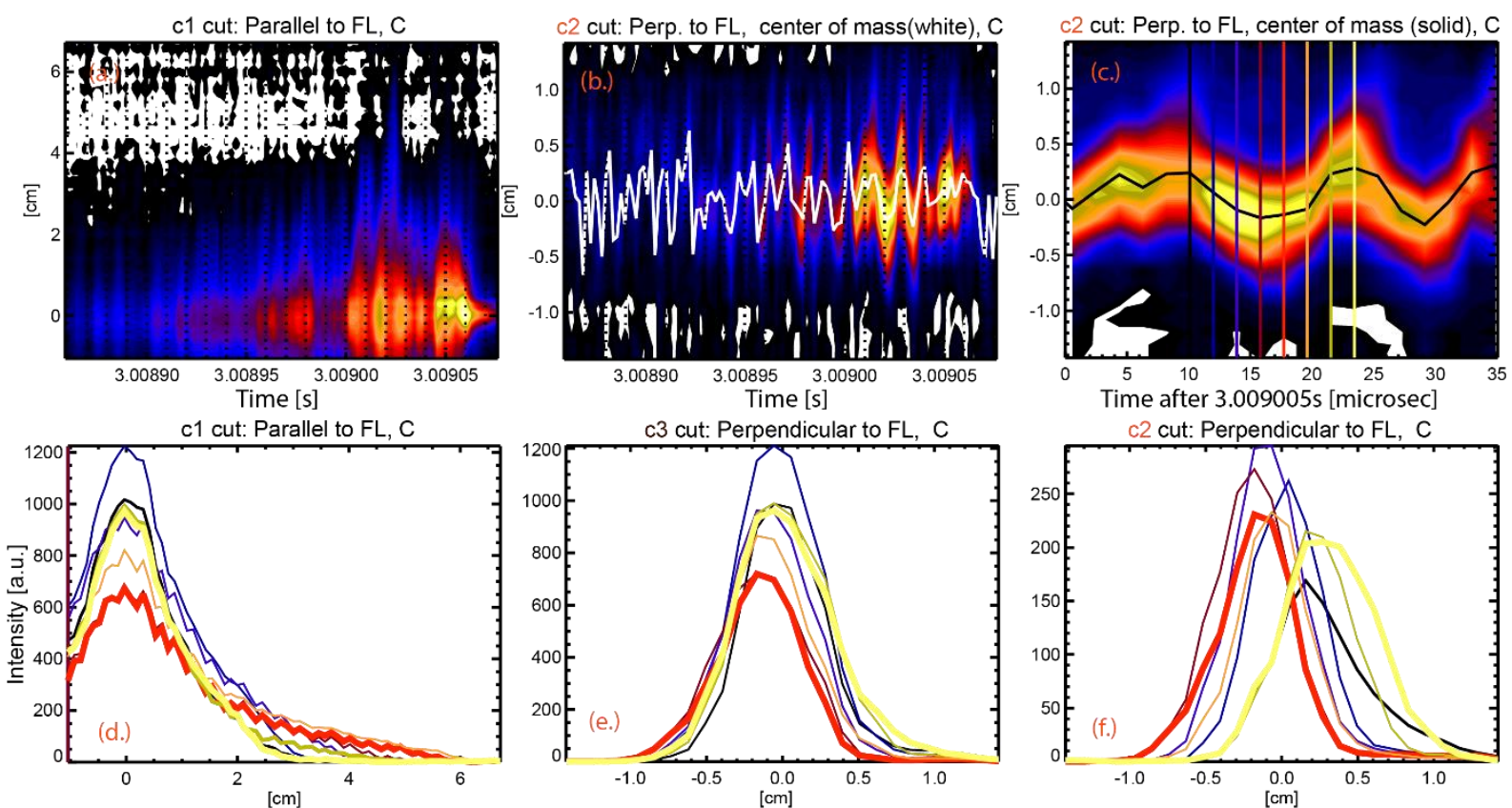

Figure 2. Radiation intensity profiles of the TESPEL cloud recorded using $\mathrm{C}$ filter as a function of time during the ablation along (a.) and perpendicular (b.) to the magnetic field line crossing the pellet for 20180927.039 discharge. The vertical axis is the distance along the cut line. On Fig 2.b the centre of mass of the profile is also over plotted (white curve). Fig2.c is a zoom of Fig2.b, the coloured vertical lines represent the time stamps of the consecutive frames (separated by about $2 \mu \mathrm{s}$ ). The individual intensity profiles at these moments are plotted on (d.)-(f.).

The TESPEL cloud has a quasiperiodic evolution with a period time of about $20 \mu \mathrm{s}$ throughout the whole ablation in the plasma. Both $\mathrm{B} \perp$ and $\mathrm{B} \|$ properties fluctuate which is associated with a vertical cloud movement and an eruption/ejection of part of the cloud (see Fig. 2 and 3). First the pellet cloud expands parallel to the magnetic field for about $10 \mu \mathrm{s}$. The neutral cloud grows slightly, while the $\mathrm{C}^{2+}$ cloud expands significantly, the location of the maximum radiation of the 
latter $\left(\mathrm{C}^{2+}\right)$ also moves further away from the pellet during the expansion. When the cloud reaches a certain $\mathrm{B} \|$ size and is becoming significantly ionised, the ionised part of the cloud (plasmoid) moves vertically (typically upwards) and detaches itself from the pellet. This takes about an additional $10 \mu \mathrm{s}$ which is equivalent to a few $\mathrm{km} / \mathrm{s}$ vertical speed. Finally, these processes are repeated. When the cloud is detached only a considerably reduced and mainly neutral cloud remains around the pellet. It is worth mentioning, that because of the view geometry, the radial ( $\operatorname{grad}(\mathrm{B})$ direction) movements cannot be observed. However, in tokamak (ASDEX Upgrade) vertical movement of the detaching pellet cloud was observed during $\operatorname{grad}(\mathrm{B})$ drift for H pellets as well [6]. Finally, similar cloud formation/drift was observed for all the magnetic geometry used during the whole campaign and also for reversed magnetic field operation.
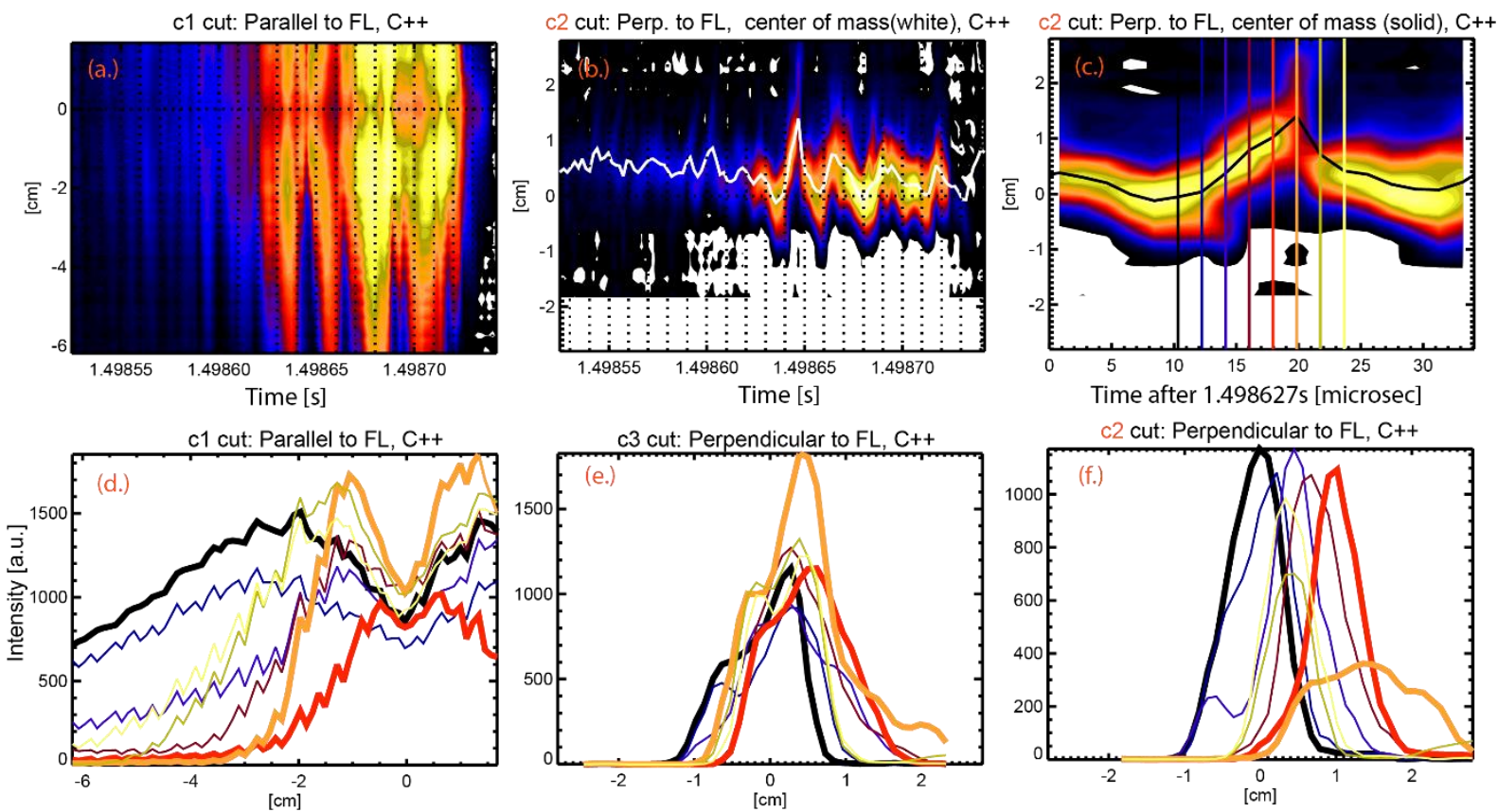

Figure 3. A set of figures similar to Fig.2, but the movie was recorded with $\mathrm{C}^{2+}$ filter for the discharge of 20180906.025 .

"This work has been carried out within the framework of the EUROfusion Consortium and has received funding from the Euratom research and training programme 2014-2018 and 2019-2020 under grant agreement No 633053. The views and opinions expressed herein do not necessarily reflect those of the European Commission."

[1] G. Kocsis et al, Europhys. Conf. Abstracts Vol. 42A, P4.1066, (2018)

[2] S. Sudo and N. Tamura, Rev. Sci. Instrum., 83, 023503 (2012)

[3] R. Bussiahn et al, Rev. Sci. Instrum., 89, 10K112 (2018)

[4] K.J. McCarthy et al, EPL, 120, 25001 (2017)

[5] G. Cseh et al, Nucl. Fusion, 57, 016022 (2017)

[6] G. Kocsis et al, Europhys. Conf. Abstracts 36F, P1.093 (2012) 